symptomatic and asymptomatic carotid artery stenosis. However, existing literature on readmission after these procedures is limited. In this study, we characterize the rate and causes of 30 and 90-day unplanned readmissions after CEA and CAS for patients with asymptomatic stenosis.

Materials and Methods Data was extracted from the Nationwide Readmission Database spanning 2010 to 2015. The patient population consisted of adult patients who underwent CEA or CAS with a primary diagnosis of occlusion and/or stenosis of a carotid artery without mention of cerebral infarction or TIA. Non-elective readmission within 30 and 90 days were identified and readmission/retreatment rates for CEA vs CAS were also compared.

Results Of 485,838 patients treated and discharged alive, $28,336(5.8 \%)$ patients were readmitted within 30 days, and $41,042(10.0 \%)$ patients were readmitted within 90 days. The most common primary diagnoses for non-elective readmission within 30 and 90 days, respectively, were cerebral artery occlusion with infarct $(3.9 \%, 3.5 \%)$, carotid artery stenosis/ occlusion without infarction $(2.8 \%, 4 \%)$, myocardial infarction $(3.7 \%, 3.5 \%)$, hematoma $(3.5 \%, 2 \%)$, TIA $(2.9 \%, 2.7 \%)$, septicemia $(2.9 \%, 3 \%)$ pneumonia $(2.9 \%, 3 \%)$ and acute kidney failure $(2.1 \%, 2.4 \%)$. The 30 and 90 day non-elective readmission rate for CEA vs CAS was $5.7 \%$ vs $6.8 \%(\mathrm{p}<0.0001)$ and $9.7 \%$ vs $12.2 \%(\mathrm{p}<0.0001)$, respectively. The 30 and $90-$ day non-elective retreatment rates for CEA vs CAS were $0.16 \%$ vs $0.20 \% \quad(p=0.0431)$ and $0.38 \%$ vs $0.50 \%$ $(\mathrm{p}=0.0001)$, respectively (figure 1$)$.

Conclusion Common reasons for 30 and 90-day non-elective readmission after CEA or CAS for asymptomatic stenosis were cerebral artery occlusion with infarct, myocardial infarction and hematoma. Rates of non-elective readmission/retreatment after 30 and 90-days were higher for CAS than CEA.

Disclosures P. Nazari: None. P. Golnari: None. R. Garcia: None. H. Weiss: None. S. Ansari: None. A. Shaibani: None. M. Hurley: None. M. Potts: None. B. Jahromi: None.

\section{0-007 AN ANALYSIS OF STROKE THROMBECTOMY INTERHOSPITAL TRANSPORTATION MODALITY}

H Dasenbrock*, A Beer-Furlan, A Vargas, J Connors, R Crowley, M Chen. Neurosurgery, Rush University, Chicago, IL

\subsection{6/neurintsurg-2019-SNIS.7}

Objective Expeditious interhospital transport of patients with potential large-vessel occlusions is key in the hub and spoke model, where patients are first taken to a local primary hospital to be evaluated for intravenous thrombolysis, and then subsequently transferred to an endovascular capable stroke center. The decision on transport modality-air versus ground transportation-may be multifactorial, dependent upon dispatch times, availability, and cost. This study aims to evaluate and quantify the presumed reduction in time to thrombectomy with air compared to ground transport.

Methods Patients undergoing mechanical thrombectomy for carotid circulation occlusion within 6 hours at an urban, comprehensive stroke center were retrospectively analyzed. Multivariable linear regression evaluated the relationship between transport modality and the time from last known well to groin puncture after adjusting for distance from the comprehensive stroke center.
Results From January 2015 to March 2018, 133 mechanical thrombectomy interhospital transfers were identified; transportation modality was air in $30.8 \% \quad(n=41)$ and ground in $69.2 \%(\mathrm{n}=92)$. The mean inter-hospital distance was 24.1 (standard deviation 16.4, range 0-62) miles. Among patients travelling greater than 10 miles, the use of air transport was associated with a significantly shorter time between last known well and groin puncture when compared to ground (by 26.3 minutes, 95\% CI: 1.1-51.9 minutes, $p=0.04)$. The benefit of air transport was greater with increasing distances, with a significantly shorter time to thrombectomy of 35.1 minutes $(p=0.02)$ if an inter-hospital distance of greater than 20 miles, and of 42.2 minutes $(p=0.03)$ if greater than 30 miles. Within 10 miles however, all patients were transported by ground.

Conclusions In this single-center analysis, helicopter emergency medical service lead to a shorter time to thrombectomy compared with ground transport. Given the known benefit to earlier revascularization on stroke outcomes, these data support the use of emergency aeromedical services when logistically feasible for stroke thrombectomy interhospital transfers greater than 10 miles.

Disclosures H. Dasenbrock: None. A. Beer-Furlan: None. A. Vargas: None. J. Connors: None. R. Crowley: None. M. Chen: 2; C; Genentech, Pneumbra, Stryker, Medtronic.

\section{0-008 HIGH-FREQUENCY OPTICAL COHERENCE TOMOGRAPHY FOR IMAGING NEUROVASCULAR IMPLANTS IN TORTUOSITY}

A Puri, M Marosfoi, G Ughi, R King, E Langan, J Chueh, M Gounis. Radiology, University of Massachusetts, Worcester, MA

\subsection{6/neurintsurg-2019-SNIS.8}

Introduction Intravascular optical coherence tomography (OCT) has an established role in the diagnosis and image guided treatment of coronary artery disease. However, due to the profile and design of the catheter, these devices are not suitable for routine application in neurointerventional surgery. We aim to demonstrate that new generation high frequency OCT (HF-OCT) can image stents and flow diverters used to treat intracranial aneurysms with acceptable workflow deployed in neurointerventional surgery.

Materials and methods An HF-OCT prototype was built (Gentuity, Sudbury MA) that is compatible with standard microcatheters (0.017'). An in vitro flow loop consisting of a patient-specific vascular replica and using porcine blood as a working fluid was deployed to test contrast infusion protocols necessary to remove blood from the target vascular section. Subsequently, eight pigs were implanted bilaterally with flow diverters and stents. The presence of acute platelet aggregation on the surface of the devices and malapposition of the device to the vessel wall was assessed by 3 raters reviewing digital subtraction angiography (DSA), high resolution cone beam CT (CBCT), and HF-OCT. Finally, using the tortuous porcine brachial artery, HF-OCT was performed, and image quality assessed.

Results In the in vitro experiment, the optimized contrast infusion protocol was delivery of contrast through the intermediate catheter (0.058' Navien, Medtronic Neurovascular, Irvine CA) delivering the HF-OCT device at a rate of $5 \mathrm{ml} / \mathrm{s}$ for 20-25 ml of contrast. This protocol resulted in complete 


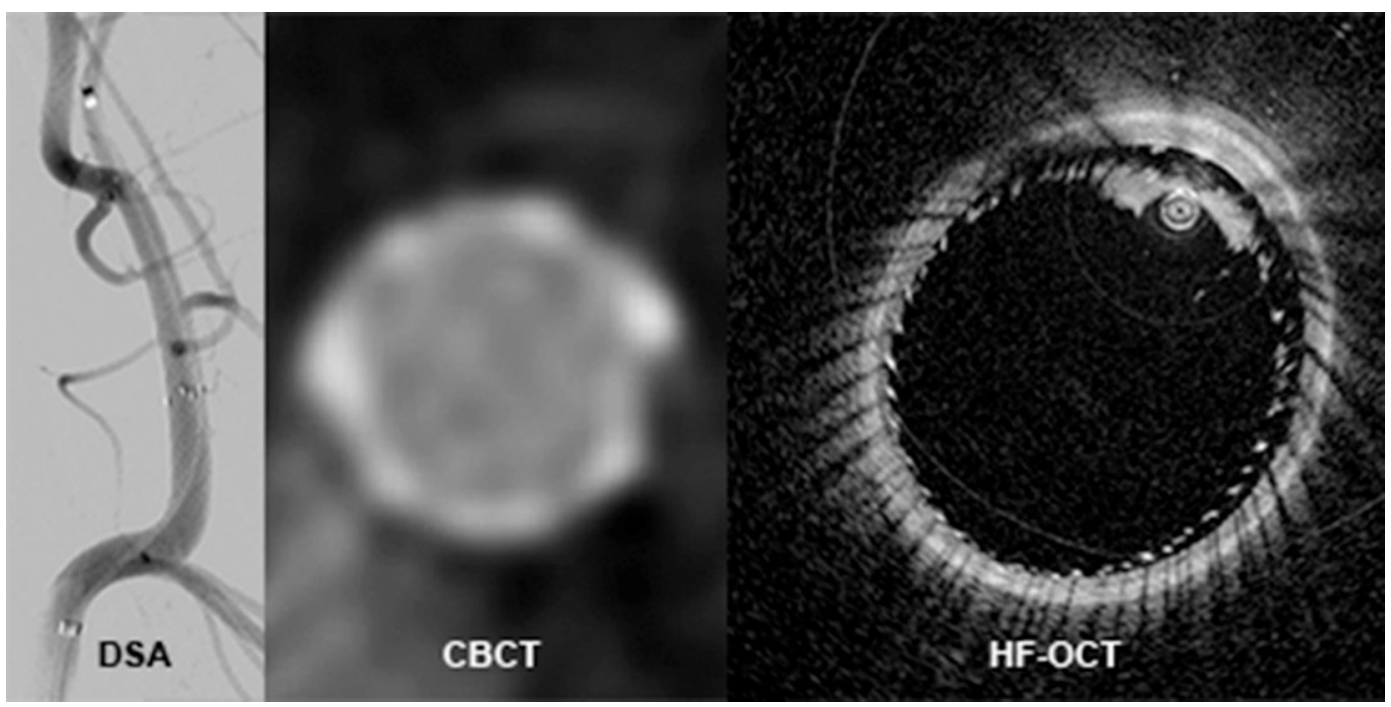

Abstract 0-008 Figure 1

clearing of highly attenuating erythrocytes for an optically clear field. In the pig study, the agreement by the reviewers (Fleiss kappa) regarding clot formation at 3 distinct locations along the flow diverter was $0.49,0.67$ and 0.90 for DSA, CBCT and HF-OCT, respectively. The agreement for diagnosing malapposition at these locations was $0.18,0.67$ and 0.87 for DSA, CBCT, and HF-OCT, respectively. In the porcine tortuosity model, in all cases, navigation and imaging was performed with uniform illumination and free from artifact.

Conclusion An HF-OCT device has been built that is compatible with neurointerventional surgery workflow and cerebrovascular anatomy. Preclinical data demonstrate consistent improvement to diagnose acute platelet aggregation and malapposition of flow diverters as compared to existing imaging modalities (figure 1).

Disclosures A. Puri: 1; C; NIH. M. Marosfoi: None. G. Ughi: 4; C; Gentuity. 5; C; Gentuity. R. King: None. E. Langan: None. J. Chueh: None. M. Gounis: 1; C; NIH.

\section{0-009 NEONATAL MRI FINDINGS ASSOCIATED WITH AGGRESSIVE EARLY CLINICAL COURSE IN NEWBORNS WITH VEIN OF GALEN MALFORMATION}

${ }^{1} \mathrm{~L}$ Arko IV*, ${ }^{2} \mathrm{D}$ Zurakowski, ${ }^{1} \mathrm{D}$ Obrach. ${ }^{1}$ Neurosurgery, Boston Children's Hospital, Boston, $M A_{;}{ }^{2}$ Anaesthesia, Boston Children's Hospital, Boston, MA

\subsection{6/neurintsurg-2019-SNIS.9}

Abstract Neonates born with vein of Galen malformations bifurcate into either (a) a cohort that experiences cardiopulmonary failure and/or early cerebral injury necessitating urgent neonatal embolization, or (b) a cohort that is clinically stable, in whom embolization can be safely deferred for months. There are no currently known imaging prognosticators that enable distinguishing these groups, and thus management is based solely on clinical factors, such as the Bicêtre criteria. In this study, MRI characteristics on DOL 1 images were evaluated in order to assess whether particular features correlated with the need for urgent neonatal intervention or neonatal death.

Methods Newborns with vein of Galen malformation who underwent DOL 1 MRI scans from 2007-2018 at a single tertiary referral center were evaluated. 18 anatomic vessel parameters were measured and statistically compared to ascertain whether any parameters correlated with neonatal death or urgent neonatal embolization (neonatal at-risk cohort, NAR) versus survival to treatment in infancy (infantile treatment cohort, IT). Vessels assessed included the median prosencephalic varix, straight/falcine sinus, sigmoid sinuses, internal jugular veins, internal carotid arteries, and basilar artery. Continuous variables were compared using the nonparametric Mann-Whitney U test. Categorical data were compared using chi-square or Fisher's exact test. Multivariable logistic regression test was applied to identify independent predictors that together can best differentiate the NAR and IT cohorts with area under the curve (AUC) used to determine predictive accuracy.

Results There were 21 patients in the neonatal at-risk cohort, and 11 patients in the infantile treatment cohort. Of the 18 variables tested, 12 were found to be significant, including: diameter and circumference of the straight/falcine sinus at its narrowest point, basilar artery diameter, internal carotid artery diameters, sigmoid sinus circumferences, and internal jugular vein diameters. Interestingly, neither the caliber (diameters/volume) nor the shape of the prosencephalic varix itself was found to distinguish the two cohorts. An occipital sinus was visualized for all patients in the NAR cohort versus $45 \%$ of the IT cohort $(\mathrm{P}<0.001)$. Starting with five significant variables by univariate analysis, multivariable regression analysis confirmed that straight/falcine sinus caliber at its narrowest, combined with large RICA diameter, provided the most predictive model (AUC $=0.870,95 \% \mathrm{CI}: 0.746-0.994, \mathrm{P}<0.001$ ).

Conclusion Based on 32 newborns with vein of Galen malformation, MRI measurements on DOL 1 were significantly different between patients at risk of neonatal death or need for urgent embolization, compared to patients in whom treatment was safely deferred until infancy. As these cohorts differ significantly in overall risk of mortality and severe neurodevelopmental morbidity, this early prognostication can be of great potential help to both families and caregivers. These findings require validation on a larger newborn population to provide evidence of generalizability.

Disclosures L. Arko: None. D. Zurakowski: None. D. Obrach: None. 